\title{
Effect of Bone Marrow Derived Mesenchymal Stem Cells on Neuropathic Pain Induced by Cisplatin In Rats
}

\section{Gulay Sezer, ${ }^{1,2}$ Arzu Yay ${ }^{3}$, Zeynep Burcin Gonen², Zeynep Soyer Sarica ${ }^{4}$, Gozde Ozge Onder $^{3}$, Berkay Saraymen ${ }^{5}$, Alan Aydin ${ }^{6}$, Emel Ozturk ${ }^{3}$, Dilek Bahar ${ }^{2}$, Yilmaz Secil $^{2}$}

${ }^{I}$ Pharmacology Department, Erciyes University, School of Medicine, Turkey, ${ }^{2}$ Erciyes University, Betul Ziya Eren Genome and Stem Cell Centre, Kayseri, Turkey, ${ }^{3}$ Histology and Embriology Department, School of Medicine, Kayseri, Turkey, ${ }^{4}$ Erciyes University, Hakan Cetinsaya Experimental and Clinical Research Centre, Kayseri, Turkey, ${ }^{5}$ Immunology Department, School of Medicine, Kayseri, Turkey, ${ }^{6}$ Anatomy Department, Faculty of Veterinary Medicine, Kayseri, Turkey

Effects of mesenchymal stem cells (MSCs) have been shown in several neurological diseases in human and several animal models. However, there is no study about the effectiveness of MSCs in chemotherapy induced neuropathy and neuropathic pain. We aimed to research the effects of bone marrow derived MSCs in neuropathic pain induced by cisplatin in rats.

Neuropathic pain was induced by injections of cisplatin and mechanical pain thresholds of rats were measured before and after dosing for several times. We transplanted different number of cells ( 1 and 5 millions) to the different groups of animals by intravenous route. Some animals injected with the fluorescence dye labeled cells to evaluate the homing. Half of the animals in groups were sacrified just 15 days after and remainings were sacrified 30 days after the MSC transplantation. We also evaluated the change of density of intraepidermal nerve fibers (IENFs) densities in foot pads of animals by immunohistochemistry. We analysed the levels of IL-1beta and TNFalpha in spinal cord (L4 and 6) samples of animals. Animals showed mechanical allodynia after the administrations of cisplatin. Antiallodynic effect was observed 10 days after the transplantation of MSCs. Moreover, 5 million MSCs transplantations reversed the reduction in density of IENFs in the paw that develops as a result of cisplatin treatment. Levels of inflammatory cytokines (TNF-alpha and IL1beta) increased in the spinal cord of animals receiving chemotherapy, while the TNFalpha levels decreased to similar levels of control in 5 million MSCs group. We observed that PKH26 labeled cells were located in the sciatic nerve and spinal cord samples with fluorescence microscopy.

In our study, we have seen that MSCs can be effective in chemotherapy-induced neuropathy in rats, especially when high numbers of cells (5 million) are administered. MSCs may have shown their effects by homing to the damaged structures and possibly by reducing inflammation through a number of factors that they secrete.

This study was supported by the Scientific and Technology Research Council of Turkey. 